\title{
Fault Diagnosis of Transformer for Auxiliary Power Supply of Photovoltaic Micro Inverter
}

\author{
Liu Hongjun, Guo Jinran, Zhang Jiyue, Yang Han \\ Department of International Education Institute, North China Electric Power University, Beijing, \\ China
}

Keywords: On-line fault diagnosis; self-organizing neural network; least squares support vector machines; Fault index

\begin{abstract}
In order to solve the problem of rapid diagnosis of fault in value-added service mode of special transformer users, based on the integration platform of measurement automation, an on-line fault diagnosis method for power supply equipment of special transformer users is designed. Firstly, the time series autoregressive model of state parameters of special transformer equipment is established, and the time series is quantized as the input value of the system by using the self-organizing neural network. Learning samples of least squares support vector machines are established based on process input in sliding time window. Then the deviation between the regression calculation results and the measured values of the eigenvectors of the model is set as the observation value. Gauss mixture model is used to fit the distribution of multidimensional observation values to build up the background model of the system. The fault index is calculated by the matching degree between the new individual observation value and the background model, thus real time diagnosis of equipment faults can be achieved. Experimental results show that this method can predict fault online quickly and accurately.
\end{abstract}

\section{Introduction}

As a key state-owned enterprise, Power Company integrates its features of public welfare, service and monopoly as a whole. Under the guidance in the construction of "one strong and three excellent " strategic objectives, frequent application of information technology, a large number of business data has been accumulated. Special transformer users have the demand of fine management and the purpose of improving the management benefit, so higher quality services are needed, consequently rapid fault detection and location is one of the focuses [1]. Due to the influence of natural environment, device aging, overload [2,3] and other reasons in the operation of power supply equipment, the performance of the equipment deteriorates gradually and even fails. Therefore, "predictive maintenance" is necessary. That is, based on the state of the equipment, maintenance mode is formed by forecasting the development trend of equipment status, so as to provide value-added services for special transformer users. At present, the threshold method is used to identify and locate the fault [4], but there are limitations and lags in this method. Due to the power supply equipment has various data types, large data and other characteristics, big data technology is introduced into equipment state detection to conduct abnormal state detection of equipment by big data analysis. But the power supply equipment abnormal detection also has some difficulties. One is how to quickly detect a small proportion of abnormal data in a large number of normal data. Another difficulty is the description of the relationship between the state parameters by using specific functions. At present, the detection space segmentation algorithm, time series model and other algorithms have some defects. In this paper, on the basis of the automatic measuring system of power supply system, data flow input is established with auto-regressive (AR) and self-organized maps (SOM). Besides, least square support vector machines (LSSVM) and Gauss mixture model (GMM) are used to calculate the prediction index of equipment fault in real time. 


\section{System overall design}

At present, Chongqing State Grid has established a measurement automation integration platform for power supply enterprise. The power supply equipment of the common transformer users and the special transformer users of the terminal layer is connected to the front collecting layer of the platform through the public communication network of the power communication network and the remote communication. Through the data exchange processing layer it enters the business processing layer for four functional management, mainly including low voltage meter reading, public transformer metering, special transformer value-added service and load management. There are demand side management and quartering line loss in the back-end integrated application layer. Through measurement integration platform, State Grid can carry out data processing and value-added services according to the information collected by special transformer equipment. The on-line fault diagnosis system in this paper is established on the basis of measurement automation platform, providing value-added services for special transformer users through big data analysis. Firstly, the state parameters are simulated by AR modeling, the data flow input is established by SOM, and the system background model is trained by calculating the observation values with SSVM, then real time computing equipment fault index is carried out for real time fault diagnosis.

\section{Establishment and quantification of state parameter model for special transformer}

\section{Autoregressive model of time series}

Time series autoregressive model is adaptable for a large number of industrial processes. Its advantage is that it has strong memory, and the value at a certain time is related to the previous time behavior. The fluctuation of the grounding current and the wire pulling force of the power supply equipment of the special transformer users is relative small, and they can be classified as stationary sequence. Environmental temperature, oil temperature and other parameters change periodically, but the range of change is small, after removing periodicity, they also belong to stationary sequence. The $A R(1)$ can be used to fit for both of them. The on-line monitoring data at t time is set as $x(t)$, the AR simulation is used to fit the state parameter model, as shown in Formula (1).

$$
x(t)=\alpha \cdot x(t-1)+e(t)=\alpha^{t} \cdot x(0)+\sum_{i=0}^{t-1} \alpha^{i} \cdot e(t-i)
$$

in which $\alpha<1, e(t) \sim N\left(\mu_{e}, \lambda^{2}\right)$ is normal distribution sequence, and $x(t) \sim N\left(\mu, \sigma^{2}\right)$ can be obtained, besides:

$$
\begin{gathered}
\mu=\mu_{e} /(1-\alpha) \\
\sigma^{2}=\left(\alpha^{2} \mu^{2}+\lambda^{2}+\mu_{e}^{2}\right) /\left(1-\alpha^{2}\right)
\end{gathered}
$$

Condition monitoring requires that each state should not exceed the specified threshold, namely, $x(t)$ at each t moment should satisfy $x(t) \in[a, b]$ and the following formula can be obtained:

$$
\begin{aligned}
a-\alpha^{k} x(t) \leq e(t+k)+\alpha e(t+k-1)+\cdots+ \\
\alpha^{k-1} e(t+1) \leq b-\alpha^{k} x(t)
\end{aligned}
$$

It can be seen from Formula (4) that if a certain threshold ${ }^{\alpha_{0}}$ exists, ${ }^{x}(t) \in[a, b]$ has got to work if and only if $\alpha<\alpha_{0}$. Due to the slow change of latent fault in power supply equipment, the monitoring data would not exceed the set threshold immediately after the device becomes abnormal. This is the defect of using the AR model to monitor the state. Therefore, further optimization model is needed. 


\section{Time series quantization}

Because the device status data does not contain tags and the amount of data is large, self-organizing neural networks can be adopted for unsupervised learning, so as to achieve quantization of time series [11]. SOM takes the entire sequence of $x(t)$ as input, and the output is $O=\left(O_{1}, O_{2}, \cdots, O_{N}\right)$. Training of $x(t)$ and the output node ${ }^{O_{j}}$ are conducted, so that the minimum distance between the two items $d\left(x(t), O_{i}(t)\right)$ can be obtained:

$$
j=i(x(t))
$$

Through correction and multiple iterations, the distance between $x(t)$ and the corresponding output node is minimized, as shown in Formula (6).

$$
\left\{\begin{array}{cc}
O_{i}(t+1)=O_{i}(t)+\beta(t)\left(x(t)-O_{i}(t)\right) & i \in N_{i}(t) \\
O_{i}(t+1)=O_{i}(t) & i \notin N_{i}(t)
\end{array}\right.
$$

in which $\beta(t) \in(0,1)$ is learning speed. The whole sequence ${ }^{x(t)}$ is transformed into discrete point time series in linear space by SOM quantization, and $O(t)$ can be expressed as:

$$
O(t)=O_{i}(x(t))
$$

\section{Design of on-line fault diagnosis}

Through AR modeling and SOM processing quantification for condition monitoring parameters of special transformer power supply equipment, a multi input system is generated. However, the relationship between on-line fault diagnosis and multiple state parameters is extremely complex. It is difficult to describe accurately by function. Moreover, the data flow is huge during the operation of the equipment. Thus the big data analysis method is used with LSSVM for rapid state classification to identify faults.

\section{LSSVM online learning}

Nonlinear mapping is used to map input data into high-dimensional feature space within LSSVM [12], then the problem of nonlinear function problem in low dimensional space is transformed into linear function estimation in high dimensional space, and the problem of regression is transformed into a constrained quadratic programming problem. For training sample set $\left(x_{i}, y_{i}\right), x_{i} \in R^{n}$, $y_{i} \in\{-1,1\}, i=1,2 \cdots, N, \quad \mathrm{n}$ is the dimension number of the sample space, $\mathrm{y}$ is a sample classification sign, $\mathrm{N}$ represents the total number of training samples, thus:

$$
\left\{\begin{array}{l}
\min \left(\frac{1}{2}\|\omega\|^{2}+\frac{C}{2} \sum_{i=1}^{n} \xi_{i}^{2}\right) \\
\text { s.t. } \omega^{\mathrm{T}} \varphi_{i}\left(x_{i}\right)+b+\xi_{i}=y_{i}
\end{array}\right.
$$

in which $\xi_{i}$ is the relaxation variable, $\omega$ is weight, $b$ is deviation, and $C$ is penalty factor. Lagrange multipliers are introduced to obtain the Lagrange function, then the kernel function is introduced, thus the regression function is obtained:

$$
y(x)=\sum_{i=1}^{n} \lambda_{i} k\left(x_{i}, x\right)+b
$$


When it is used for on-line modeling of time-varying processes, sliding time window process parameters are used to establish learning samples. The time window F shall not be too long due to the dynamic performance requirement, and incremental learning algorithm should be adopted. The sample set $\left\{\left(x_{i}, y_{i}\right)\right\}_{i=1}^{i=l}$ expands gradually over time, and the LSSVM regression function parameters of $a, b$ and the kernel matrix $Q$ change accordingly. The renewal of linear equation is:

$$
\left[\begin{array}{cc}
0 & e 1^{T} \\
e 1 & Q(l)+C^{-1} I
\end{array}\right] \cdot\left[\begin{array}{l}
b(l) \\
a(l)
\end{array}\right]=\left[\begin{array}{c}
0 \\
y(l)
\end{array}\right]
$$

In which $^{e 1=(1,1, \cdots, 1)^{T}}$, and the solution is:

$$
\begin{array}{r}
b(l)=\frac{e 1^{T}\left(Q(l)+C^{-1} I\right)^{-1} y(l)}{e 1^{T}\left(Q(l)+C^{-1} I\right)^{-1} e 1} \\
a(l)=\left(Q(l)+C^{-1} I\right)^{-1}(y(l)-e 1 \cdot b(l))
\end{array}
$$

The obtained regression function is:

$$
y(x, l)=\sum_{i=1}^{l} a(l) \cdot k\left(x_{i}, x\right)+b(l)
$$

The process of calculating regression function is mainly changed into calculation of $Q(l)+C^{-1} I$, when new samples $\left\{\left(x_{l+1}, y_{l+1}\right)\right\}$ are added, we have:

$$
Q(l)+C^{-1} I=\left[\begin{array}{cc}
Q(l)+C^{-1} I & M(l+1) \\
M(l+1) & m(l+1)
\end{array}\right]
$$

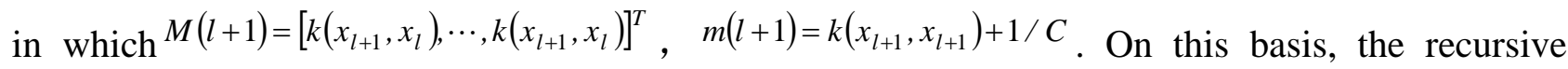
calculation of $H(l)^{-1}$ can be done by using the inverse formula of partitioned matrix, and the online learning algorithm is completed.

\section{State recognition algorithm}

Research shows that when the system state is abnormal, the LSSVM regression results and eigenvectors will have a large deviation of observation value. Its calculation formula is shown as Formula (15).

$$
\sigma_{j}=\left[\sqrt{\left(a_{1}-a_{1}^{\prime}\right)^{2}}, \cdots, \sqrt{\left(a_{m}-a_{m}^{\prime}\right)^{2}}\right]
$$

in which $m$ is the vector dimension number of the system observation vector, $a_{i}$ is the $i$ th variable measured value of the eigenvector ${ }^{y}, a^{\prime}$ is the output eigenvalues for the corresponding LSSVM model. In the early stage of fault, most of the measured values are normal data, outliers take up a small proportion and rarely exceed the theoretical threshold. Therefore, the idea of eliminating background detection in current image processing is introduced [13]. The normal state is set as the fault background after its identification, so as to identify subtle changes in the early stages of the fault. The probability of occurrence of the observation value $x_{i}$ at the moment $i$ is defined as the weighted sum of probabilities of the corresponding Gauss distribution values. That is: 


$$
p(x \mid \Theta)=\sum_{k=1}^{K} w_{k, i} N_{k}\left(x \mid \mu_{k}, C_{k}\right)
$$

in which ${ }^{x=}\left[x_{1}, x_{2}, \cdots, x_{m}\right]_{\text {is }}$ the column vector of observation in m dimension, $\Theta=\left[w_{k}, \mu_{k}, C_{k}\right]_{\text {is }}$ the set of all parameters, $N_{k}\left(x \mid \mu_{k}, C_{k}\right)$ is the kth Gauss distribution, the average value of $N_{k}\left(x \mid \mu_{k}, C_{k}\right)_{\text {is }} \mu_{k}$, and the variance is $C_{k},{ }^{w, i}$ is the prior probability of $x_{k} \in N_{k}\left(x \mid \mu_{k}, C_{k}\right)$. If the specimen $X=\left\{x_{1}, x_{2}, \cdots, x_{N}\right\}$ has a likelihood function of $L(\Theta \mid X)$, then there is:

$$
\log (L(\Theta \mid X))=\sum_{j=1}^{N} \log p\left(x_{j} \mid \Theta\right)
$$

In the initial operation, each initial value of $C_{k}$ in $N_{k}\left(x \mid \mu_{k}, C_{k}\right)$ is a unit matrix, $w_{k, i}=1 / K$, $\mu_{k}$ is the first observation value. The prior probability of the new observation ${ }^{x_{k}} \in N_{k}\left(x \mid \mu_{k}, C_{k}\right)$ is obtained:

$$
p\left(k \mid x_{j}, \Theta^{h}\right)=\frac{w_{k} N_{k}\left(x_{j}, \Phi^{h}\right)}{\sum_{k=1}^{K} w_{k} N_{k}\left(x_{j}, \Phi^{h}\right)}
$$

in which $\Phi^{h}$ is the old parameter. The mean ${ }^{\mu_{k, t-1}}$, covariance matrix ${ }^{C_{k, t-1}}$ and prior probability $w_{k, t-1}$ are updated respectively according to the observation values. Repeat iteration until Formula (19) is satisfied:

$$
\left|\log \left(L\left(X \mid \Phi^{h}\right)\right)-\log (L(X \mid \Phi))\right|<\varepsilon
$$

in which $\log \left(L\left(X \mid \Phi^{h}\right)\right)$ is calculated by Formula (17), ${ }^{\varepsilon}$ generally does not exceed $10^{-5}$, and $\operatorname{lo} g(L(X \mid \Phi))$ is the calculation value after the parameter is updated.

In order to provide system background information, $\mathrm{K}$ distributions are put in order according to size of $w_{k} /\left\|\sigma_{k}\right\|^{2}$.Among them $\sigma_{k}$ is the standard deviation of the kth distribution, and $w_{k}$ is the weight for the elements of the kth distribution. The prior $C$ distributions with the total cumulative probability more than $T$ are set as the background system, $C$ should be in accordance with Formula (20):

$$
C=\arg \min _{C}\left(\sum_{k=1}^{C} w_{k}>T\right)
$$

in which $T$ is the probability of any observation value for the system belonging to the background system. It is actually the global prior probability. After identifying the background of the system, the fault is identified, namely the new individuals appearing on the background system are found. First, the new individual observation value ${ }^{x_{t}}$ is judged whether it matches with the background, i.e., the existing $K$ Gauss distributions:

$$
\left\{\begin{array}{c}
d_{k, t}^{T} d_{k, t}<\lambda^{2} \\
d_{k, t}=\left(\sigma_{k, t} I\right)^{-1}\left(x_{t}-u_{k, t}\right)
\end{array}\right.
$$

in which $\lambda$ is constant, and the range of value is $[2,3]$.

If $x_{t}$ matches the background, the mean, covariance matrix and prior probabilities are updated according to the new calculated observation values respectively, while the variance and expected value remain unchanged, And the rest Gauss model weights become smaller. Then the fault index $I_{F P}$ is updated as follows: 


$$
I_{F P}=\frac{1}{\alpha} \sum_{t=1}^{\alpha}\left(-\ln \left(\sum_{k=1}^{n} w_{k} N_{k}\left(x_{t} \mid k ; \mu_{k}, C_{k}\right)\right)\right)
$$

in which $\alpha$ is sliding window for reduction of misjudgment rate. The greater the value, the higher the weight of historical value is. After that, the judgment of new observation value is repeated.

\section{Experimental verification}

In this paper the $220 \mathrm{kV}$ transformer of a special transformer in Yongchuan District of Chongqing city is selected to set up the manmade hidden trouble for the system performance test. In order to reduce the influence of power rationing factors, the power supply equipment is close to the power supply center with a distance of about 3.4 kilometers. Data is transferred through power communication network. The main parameters settings of the system are listed in Table 1.

Tab. 1 Main parameter settings

\begin{tabular}{ccc}
\hline No. & Parameter name & Parameter value \\
\hline 1 & $\mathrm{C}$ & 30 \\
2 & $\beta^{2}$ & 9 \\
3 & $F$ & 5 \\
4 & $\alpha$ & 0.8 \\
5 & $\mu_{\varepsilon}$ & 0 \\
6 & $\lambda$ & 0.01 \\
7 & $\mathrm{~K}$ & 15 \\
8 & $\mathrm{~T}$ & 0.7 \\
9 & $\varepsilon$ & $7 \bullet 10^{-6}$ \\
10 & Sampling period & $1 \mathrm{~s}$ \\
\hline
\end{tabular}

Transformer load, oil temperature, environmental temperature and other real-time data are taken as monitoring objects. Taking load as an example, the prediction error of the observation and real values is shown in Figure 1. As shown in the figure, after 55 sampling periods, the prediction accuracy of LSSVM model reaches a high level.

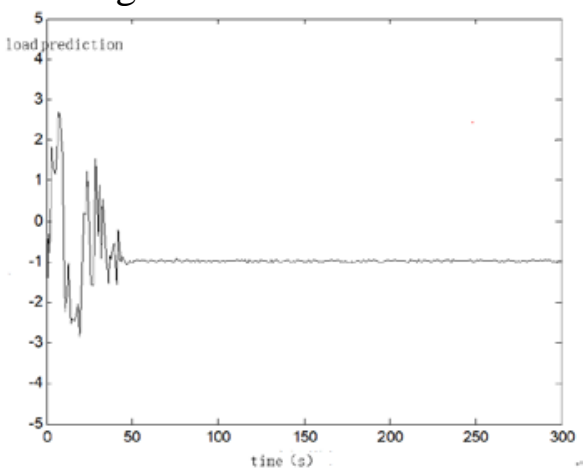

Fig. 1 The error of load prediction

In order to detect the sensitivity of system fault prediction, transformer load abnormity is set manually. In order to improve safety, the fault setting process is operated at the input terminal of the model. Load rate is improved through program to $[75 \%, 85 \%]$. Long term operation will cause transformer fault under this condition. The resulting fault index is shown in Figure 2. From the moment of increasing load, the fault index is gradually increasing. After about 14 seconds, the fault index began to increase significantly. Although there is a certain fluctuation in the process, but the overall has an upward trend. The abnormal state of the load maintained about 90 minutes, then the transformer reported the fault, while the system could reflect the abnormal state of equipment in 14 seconds according to the fault index. It is proved that the fault prediction of the system has high sensitivity. It is obvious that the fault can be quickly detected and located according to the fault index. This kind of predictive fault location is superior to the traditional single equipment fault self-checking system in economic cost and time cost. It reflects the timeliness of fault on-line diagnosis method based on measurement automation integration platform. 


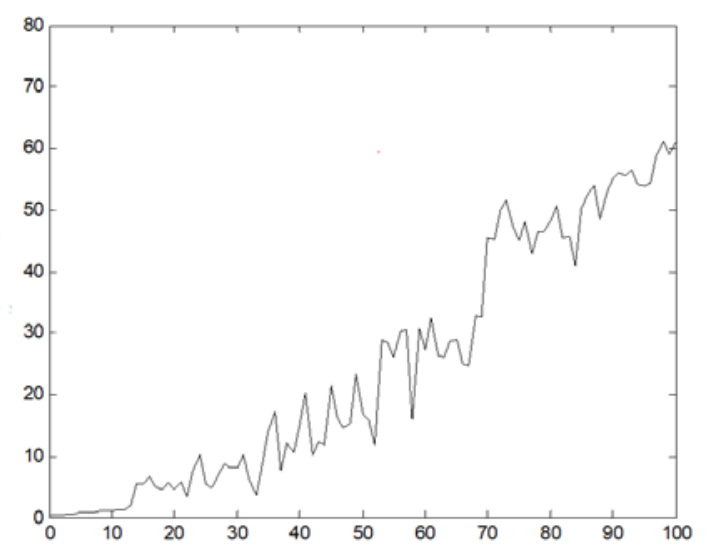

Figure 2 Calculation results of fault index

\section{Conclusions}

In this paper, an automatic fault detection method for power supply equipment of special transformer users is designed based on the existing measurement automation integration platform. By monitoring the load, oil temperature, environmental temperature and other real-time data of power supply equipment, the observation values are generated by the regression computational results and the measured values of least squares support vector machines. On-line fault index diagnosis is achieved based on matching degree between new observation value and background model. The health status can be predicted before the equipment fails, and its sensitivity and timeliness have been verified. The next step is to expand the system monitoring state parameters and monitoring algorithm to improve the system model.

\section{References}

[1] Weisen Pan, Shizhan Chen, Zhiyong Feng. Automatic Clustering of Social Tag using Community Detection. Applied Mathematics \& Information Sciences, 2013, 7(2): 675-681.

[2] Yingyue Zhang, Qi Li, William J. Welsh, Prabhas V. Moghe, and Kathryn E. Uhrich, Micellar and Structural Stability of Nanoscale Amphiphilic Polymers: Implications for Anti-atherosclerotic Bioactivity, Biomaterials, 2016, 84, 230-240.

[3] Jennifer W. Chan, Yingyue Zhang, and Kathryn E. Uhrich, Amphiphilic Macromolecule Self-Assembled Monolayers Suppress Smooth Muscle Cell Proliferation, Bioconjugate Chemistry, 2015, 26(7), 1359-1369.

[4] Dalia S. Abdelhamid, Yingyue Zhang, Daniel R. Lewis, Prabhas V. Moghe, William J. Welsh, and Kathryn E. Uhrich, Tartaric Acid-based Amphiphilic Macromolecules with Ether Linkages Exhibit Enhanced Repression of Oxidized Low Density Lipoprotein Uptake, Biomaterials, 2015, 53, 32-39. 